\title{
Inverse dynamic model of the pupil muscle plant in the simulation of response to sound, stimuli and hippus
}

\author{
E. Suaste Gómez \& H. Reyes Cruz \\ Centro de Investigación y de Estudios Avanzados del Instituto Politécnico \\ Nacional/Depto. Ingeniería eléctrica Sección Bioelectrónica, México
}

\begin{abstract}
The model developed by Usui and Hirata was used to simulate the pupil response to a light stimulus based on autonomic nervous activity, such as sympathetic and parasympathetic activity. This model was recreated in SIMULINK finding that it did not consider the limitations of the pupil in frequency which Stark was reported. The pupil acts as a lowpass filter with a cut-off frequency of $1.5 \mathrm{~Hz}$ approximately. These conditions are generated by the unidirectional rate sensitivities. Then, considering this fact, the original model by placing the output of the lowpass filter of $-20 \mathrm{~dB} /$ decade was modified, which there certain changes are regarding the response of the model by filtering high frequencies, and a delay by the filter.

To obtain the pupil dynamic response induced by sound stimulation, the hippus and flickering of practice mode was using conventional (NTSC) and high speed videoculography thus, to determine sympathetic and parasympathetic activity, in order to recreate such behaviour the Hirata-Usui model was used with a few modification.
\end{abstract}

Keywords: pupil dynamic, Hirata-Usui's model, autonomic nervous.

\section{Introduction}

For several years the eye has been an organ of great interest to study, given that it can communicate with the environment by getting information of all kinds, from the fact finding food, to travel without suffering from accidents, getting away from everything that may affect, to name a few. Hence the importance of this is to carry out our daily chores. 
The autonomic nervous system (ANS) controls some functions of the eye such as pupil diameter. The pupillary dynamics is used as a non-invasive tool in the study of the ANS $[1,2]$.

Hence, the interest of using the Hirata-Usui's model, where the first part recreates the model, then we make changes that we consider relevant to the model and finally observed the ability of the model to recreate the dynamic of the pupil to the sound stimulus, hippus and blinking.

In the present chapter uses the reflection of fight/flight [1, 2] or also known as fight/flight response as a means to activate the ANS and obtain the activity of it through the pupillary area. For that purpose, sound stimuli are sent to the person during the study, which would cause thereby activating startle fight/flight response by the ANS.

On the other hand, there is the phenomenon of hippus [3], where the pupil moves even if they receive a stimulus of light, a phenomenon that way considering the innervations by the ANS in the pupil, can be attributed to fact that there is an unbalance by the sympathetic and parasympathetic system.

A response was analyzed but was behaving as the pupil to a flicker, which are thrown over the controls and processes of the pupil to light, however can provide information on pupillary dynamics and activity of ANS.

Finally, seen such processes simulated by a Hirata-Usui model in which you can use the sympathetic and parasympathetic activity to obtain thereby the dynamics of the pupil to react when each of the stimuli described above.

\section{Hirata-Usui model}

The model was designed considering the innervations of ANS in muscles that governing the pupil diameter, the sphincter and dilator and based on the physiological experimental results describing the behaviour of these muscles in terms of its viscoelasticity and tension generated. Elasticity, viscosity and tension of muscle were described by the following equations:

Elasticity:

$$
\mathrm{P}_{\mathrm{p}}=\left\{\begin{array}{cc}
\mathrm{a}\left(\mathrm{x}-\mathrm{l}_{0}\right)^{4}+\mathrm{b}\left(\mathrm{x}-\mathrm{l}_{0}\right)^{2}, & \mathrm{x} \geq \mathrm{l}_{0} \\
0, & \mathrm{x}<\mathrm{l}_{0}
\end{array}\right.
$$

where $a$ and $b$ are constants, and $l_{0}$ is the muscle length at the rest.

Viscosity:

$$
P_{v}= \begin{cases}D_{+} \frac{d x}{d t}, & \frac{d x}{d t} \geq 0 \\ D_{-} \frac{d x}{d t}, & \frac{d x}{d t}<0\end{cases}
$$

where $D_{+}$and $D_{\text {- }}$ are viscous coefficients at the phase of stretch and release, respectively.

Tension:

$$
\begin{gathered}
\mathrm{P}_{\mathrm{a}}=\mathrm{g}(\mathrm{t}) \mathrm{p}_{\mathrm{a}}(\mathrm{x}) \\
\mathrm{p}_{\mathrm{a}}(\mathrm{x})=\left\{\begin{array}{cl}
\mathrm{P}_{0}-\mathrm{c}\left(\mathrm{x}-\mathrm{L}_{0}\right)^{2}, & \mathrm{P}_{0} \geq \mathrm{c}\left(\mathrm{x}-\mathrm{L}_{0}\right)^{2} \\
0, & \mathrm{P}_{0}<c\left(\mathrm{x}-\mathrm{L}_{0}\right)^{2}
\end{array}\right.
\end{gathered}
$$




$$
\begin{gathered}
g(t)=g_{\text {stat }}+\hat{g}(t) \\
g_{\text {stat }}=\gamma E_{\text {stat }} \\
\frac{d^{2} \hat{g}(t)}{d t^{2}}+(2 \alpha+\beta) \frac{d \hat{g}(t)}{d t}+\alpha(\alpha+\beta) \hat{g}(t)=\beta \widehat{E}\left(t-t_{D}\right)
\end{gathered}
$$

where $c$ and $\gamma$ are constants. $L_{0}$ is the muscle length at which the maximum active tension $P_{0}$ is produced $\alpha$ and $\beta$ are the time constants of the off and onslope of the isometric twitch response, respectively $g(t)$ and $p_{a}(x)$ are inputs dependent and muscle length dependent terms of active tension, respectively $g_{\text {stat }}$ is a DC part of $g(t)$, while $\hat{\mathrm{g}}(\mathrm{t})$ represents a $\mathrm{AC}$ part $t_{D}$ is the delay time of response $[4,5]$.

To obtain the full model was necessary to combine the action of the dilator and sphincter according to the structure of the pupil. Hirata and Usui simplified the two dimensional plant structures into the one dimensional push-pull structure as it showed in Figure 1.
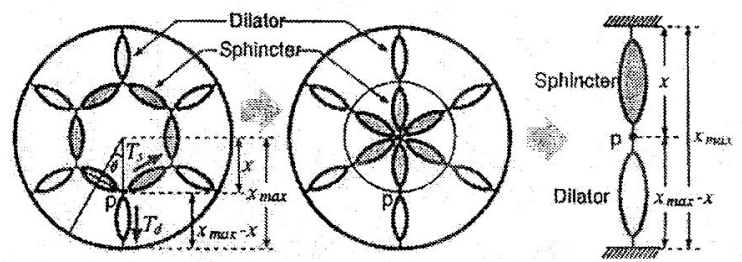

Figure 1: Mechanical model used by Hirata and Usui.

Finally, the model is a differential equations system with 13 equations that simulate the behaviour of the pupil depending on the sympathetic and parasympathetic activity, which has been used to simulate the behaviour to a light stimulus, in our case was used to simulate the pupil response to sound stimulation, the hippus and flicker.

\section{Fight/flight response}

Anxiety is a response to danger or threat. Scientifically, the short-term anxiety response is called fight/flight. This is because, all effects are designed to fight, or avoid danger. Therefore, the first purpose of anxiety is to protect the human body $[1,6]$.

The response of fight/flight generates immediate changes in the body that allow the man a better and quicker response to danger. These changes are mediated by activation of the sympathetic nervous system and these effects are $[1,6]$ :

1. Pupils dilate, to increase the visual field.

2. Heart rate and force of contraction is increased and blood pressure also increases their values to carry nutrients and energy to the muscles preparing them for flight. 
3. Blood vessels in the skin and viscera constrict.

4. The remainder of the blood vessels dilate. This causes a faster blood flow of blood to the dilated blood vessels in skeletal muscle, heart and lungs, organs involved in combating the danger.

5. Rapid breathing and deeper and bronchioles dilate to allow more rapid movement of air into and out of the lungs.

6. The contractions of blood sugar increases as the liver glycogen are converted to glucose for energy.

7. The core of the adrenal glands is stimulated to produce adrenaline and noradrenaline, hormones that enhance and prolong sympathetic effects.

8. The processes are not essential to meet stressful situations are inhibited. For example, the muscular movements of the digestive tract and secretions are slower or even come to a halt.

\section{Development}

For comprise the different pupil responses mentioned (flicker, hippus and sound stimuli), were performed an experimental tests on patients while on its right eye was recording by a high speed camera (DALSA) brand. Infrared and a superbright white LED were used to illuminate the eye-recorded. Infrared LEDs were employed to find a greater contrast between the pupil and the iris in the images taken from high speed camera to improve digital processing. The white LED current was controlled to supply a constant luminance of $1000 \mathrm{~cd} / \mathrm{m}^{2}$ to prevent the pupil reach the maximum contraction or expansion.

To obtain hippus and sound stimuli pupil responses, the test staring after the patient had been looking the camera for $1 \mathrm{~min}$, then during 10 seconds of capture in which patient was informed that he should not blink. In random manner, sound stimulus is sent to him. The stimulus was a mixture of two different types of music or pure tones. Sound stimulus triggers the fight/flight response and thereby activates the autonomic nervous system [1] which is observed as changes in the pupil diameter. After $10 \mathrm{~s}$, the patient was informed that can do a flick if he wants, in that moment begin the part of the test to record flicker response. This process avoid the patient were forced to flick that could disturb results. Recorded images were processed in Vision Builder 3.5 to obtain corresponding pupilograms. Relationship adjusts between the pixels and distances were done before each test to ensure that images distances corresponded to real proportions. Afterwards, pupillary area was framing and filtering to smooth the pupil. Changes in brightness and contrast were also made to highlight the pupil. Finally a thresholding process to detect the pupil had been taken place to calculate pupillary area. This method is shown in Figure 2.

Experimental result from previous test lead up to the model developed in SIMULINK of Matlab as shown on Figure 3 [5]. 


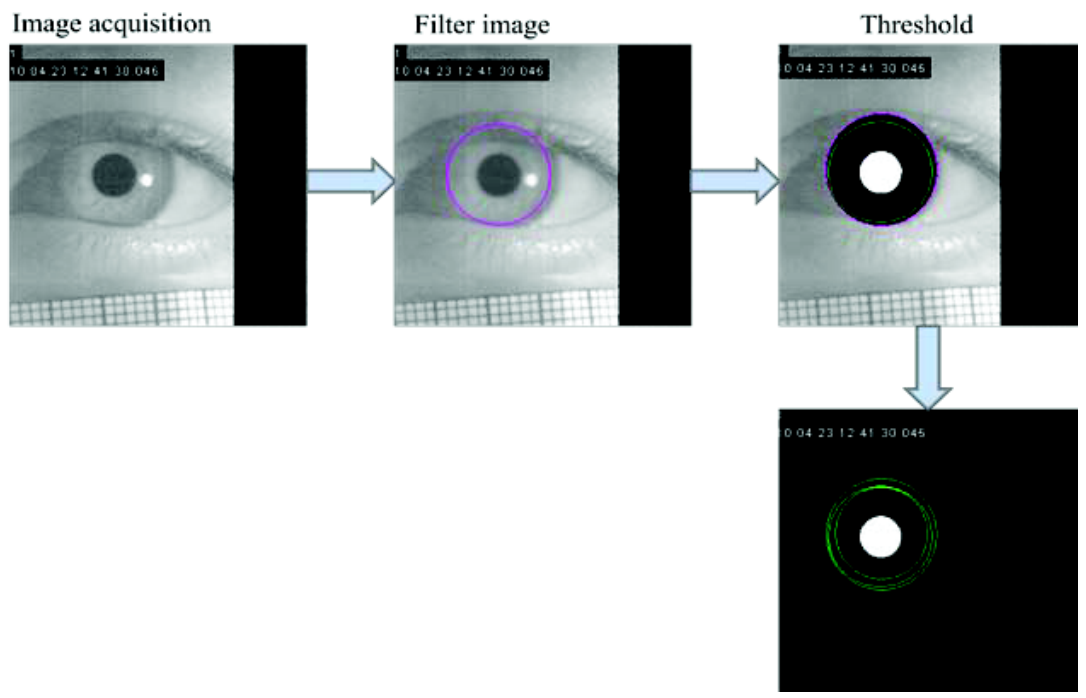

Figure 2: $\quad$ Steps for image processing.

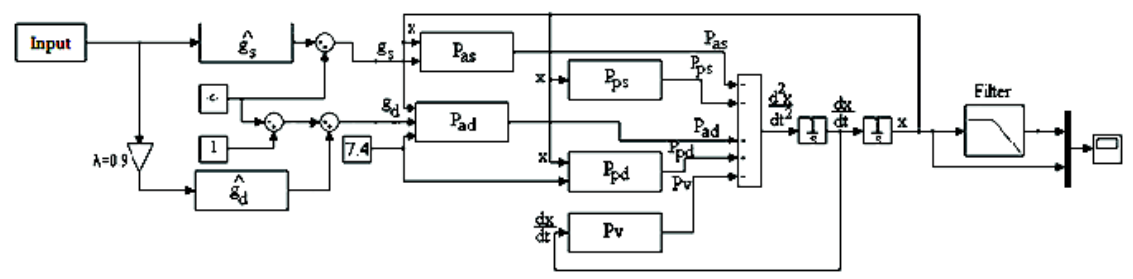

Figure 3: Development of Hirata's model in SIMULINK.

\section{Results}

The pupillary response that was observed in sound stimuli test was a reduction followed by a dilation of the pupil area which can be seen in Figure 4.

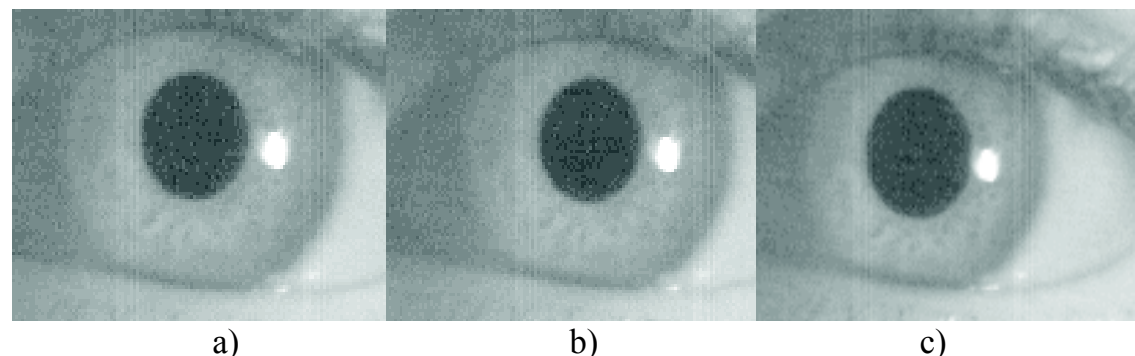

Figure 4: Images of the pupil response. a) Before of the sound stimuli b) when send the sound stimuli c) After of the sound stimuli. 
The complete response is shown in Figure 5. From Figure 5, high level pulse corresponds to the activation of the stimulus, low state corresponds to the stage where the person listens to quiet music, the edge where the pulse changes its state corresponds to the part in which person listening to loud music and filtered in this case $600 \mathrm{~Hz}$, resonant frequency of the membrane charge to convert sound into electrical impulses for registration and processing in the nervous system, from that point as there is observed a decrease in area pupil, followed by a further increase in the same.

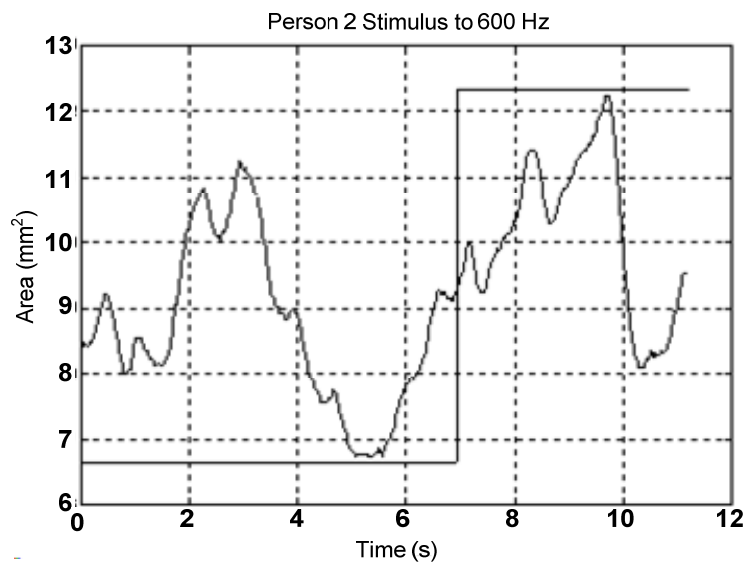

Figure 5: $\quad$ Example of pupil dynamics for acoustic stimulation.

On the other side can also observe the presence of hippus by the absence of one steady pupillary area. Otherwise, we had not been excepted pupil response in the time period where the subject listen to classical music, but the pupil response in the subject to it were very similar when he listening to loud music. In the case of pure tones similar behaviour was observed where the stimulus before and after hippus note tone, a contraction followed by expansion.

In the case of hippus there is a particular behaviour cause for the same person there are different answers as shown in Figure 6 where being the same person without a change in the pupillary dynamics.

Referring to flicker was observed behaviour similar to that displayed in the sound stimuli, i.e. a contraction shortly before and after the blink here is a dilation of the pupil.

Once developed the model of Hirata-Usui, a filter was added to the exit of the purpose of simulating the frequency limitations of the eye, considering a cut-off frequency of $1.5 \mathrm{~Hz}[7,8]$. Since the filter implemented in the model simulation was continued to the previous answers which resulted in the graphs shown in Figure 7.

In Figure 7a) noted as the model is able to recreate the pupillary response to sound stimulation as can be seen in the first instance the contraction and dilation after her. 

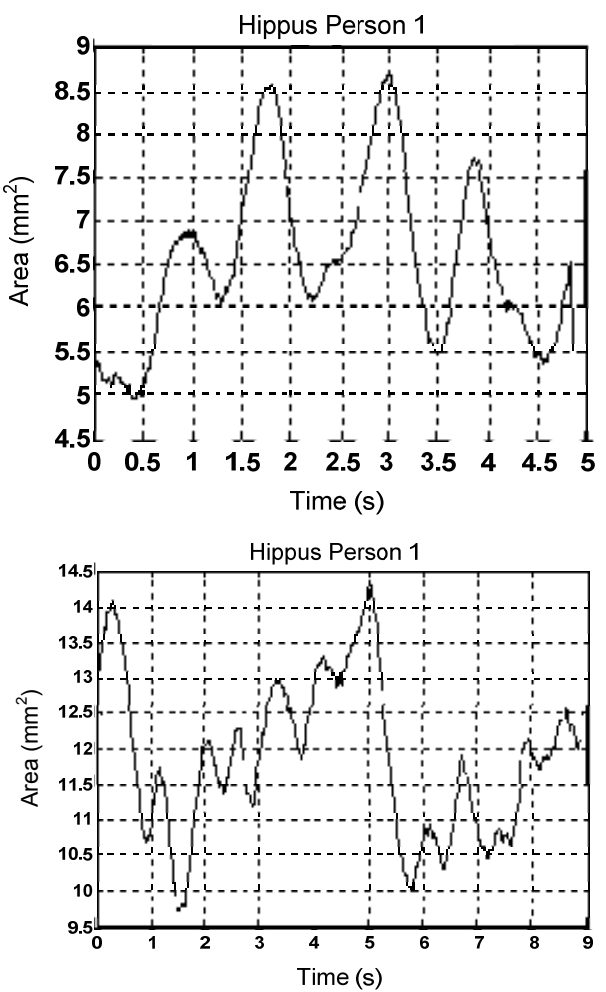

Figure 6: Hippus of the same person and under the same light illumination intensity.

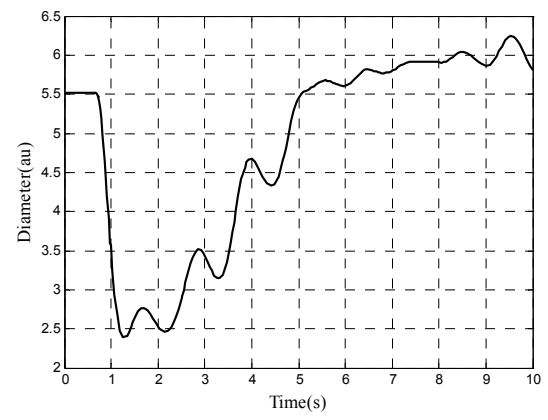

a)

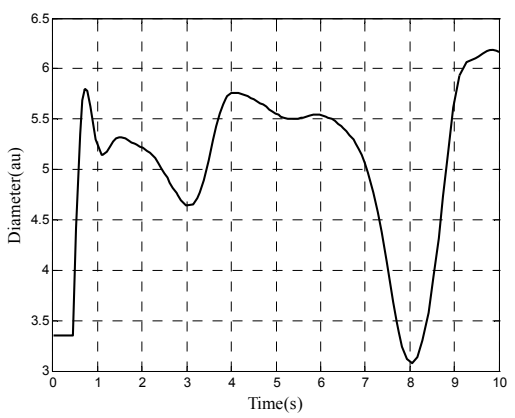

b)

Figure 7: Simulation of: a) the pupillary response to a sound stimulus and a flicker noise b) hippus.

In the case of hippus, to get a random process that results in a random response were used different input to determine the ability of the model. The input was a sine wave with amplitude of $1 \mathrm{~V}$ and frequency of $0.5 \mathrm{~Hz}$ which 
obviously not contain the random part sought. Other entries were random numbers considering a mean 0 and variance 1 and the other entire was white noise, both generated by the software Matlab. These last two options will provide a better result to generate the random process.

\section{Conclusions}

There is a response from the pupil to sound stimuli which can see initially as a contraction. The first instance activates the parasympathetic system, however after this, there is a noticeable expansion of greater magnitude by activating the sympathetic system. In some cases the contraction is not noticeable, but in all cases the dilation is noted that for all sound stimuli will activate the parasympathetic system. The response observed during blinking is similar to the response of sound stimuli.

The hippus shows us all the time the pupil is moving because they never achieved complete stability by the sympathetic and parasympathetic system.

Hirata and Usui's model is able to simulate the eye's response to stimuli above, the adjustment itself to keep the simulation within the frequency range of the human eye.

\section{References}

[1] G. J. Tortora \& S. R. Grabowski; Anatomy and physiology principles, Oxford, Mexico, 1998.

[2] Arthur C. Guyton, John E. Hall; Medical physiology tractate, McGraw Hill, Mexico, 2002.

[3] Clynes M., The non-linear biological dynamics of unidirectional rate sensitivity illustrated by analog computer analysis, pupillary reflex to light and sound, and heart rate behavior, Annals New York Academy of Sciences, Vol. 98, pp. 806-845, 1962.

[4] Hirata Y. \& Usui S., Nonlinear dynamical model for human papillary muscle plant, IEICE J77-DII, pp. 170-180, 1994.

[5] Usui S. and Hirata Y., Estimation of autonomic nervous activity using the inverse dynamic model of the pupil muscle plant, Annals of Biomedical Engineering, vol. 23, pp. 375-387, 1995.

[6] Villamar L. \& Suaste E., High velocity videoculography to determination of the pupil dynamics Tenth Symposium on Medical Physics-2008, edited by G.H. Corral and L. M. Montaño, AIP Conf. Proc. 1032, Melville, New York, pp. 276-279, 2008.

[7] Stark L., Neurological controls systems studies in bioengineering, University of California Berkeley, Plenum press, New York, 1968.

[8] Clynes M., Unidirectional rate sensitivity: a biocybernetic law of reflex and humorai, systems as physiologic channels of control and communication, Annals New York Academy of Sciences, vol. 92, pp. 946-969, 1961. 